\title{
Urgences
}

\section{Roman et poésie : notes}

\section{Fernand Ouellette}

Numéro 28, mai 1990

Le roman comme poétique

URI : https://id.erudit.org/iderudit/025593ar

DOI : https://doi.org/10.7202/025593ar

Aller au sommaire du numéro

Éditeur(s)

Urgences

ISSN

0226-9554 (imprimé)

1927-3924 (numérique)

Découvrir la revue

Citer cet article

Ouellette, F. (1990). Roman et poésie : notes. Urgences, (28), 108-110.

https://doi.org/10.7202/025593ar d'utilisation que vous pouvez consulter en ligne.

https://apropos.erudit.org/fr/usagers/politique-dutilisation/ 


\section{Roman et poésie: notes Fernand Ouellette}

Je suis entré dans mon premier roman sous la force d'une poussée qui devait m'aider d̀ sortir d'une impasse dans ma vie ou dans ma quête. En ce sens, Tu regardais intensément Geneviève est un fragment d'"autofiction", ou, comme je l'ai ecrit, un autoportrait de couple. Mais ce projet n'en demeure pas moins romanesque dans son élaboration, sa composition, sa narration, bref, dans son travail de l'imaginaire.

Dans mes deux autres romans, je me suis risqué dans des zones plus insaisissables de ma relation avec la vie, les hommes et le monde. Et, sans ces expériences de narration, je n'aurais sans doute jamais écrit Les heures dans une forme poétique que hantent quelque peu l'arrière-pensée du récit et le suspens.

Au début de Geneviève, par exemple, je ne me suis avancé dans l'ecriture qu'en étouffant d'une façon aveugle le langage. Je glissais vers un niveau d'écriture qui ne faisait qu'anémier ma prose. Je sous-écrivais, trop obséde par la crainte de glisser dans l'écriture poétique, ce dont je me méfiais le plus. Je m'enfonçais si directement dans la brutalité des choses que j'en perdais toute lumière. Je ne parvenais plus à émerger de la matière, alors que, jusqu'd cette experience nouvelle, j'avais toujours cuuré dans le langage comme dans un espace de transfiguration. En somme, je traînais de l'aile, surtout dans les transitions que je croyais nécessaires a l'évolution de l'intrigue. Et s'étiolait la magie operante qui soulève la matiere verbale, qui lézarde l'opacité, qui relie dans un lien subtil le non-dit, l'obscur, l'absent d l'indicible, au présent de l'ailleurs, au lumineux, ici, parmi nous. C'est peut-être pour cette raison que je me suis menagé des plages de prose poétique qui avaient moins une fonction de raccord que la puissance de suggerer une tonalité ou une atmosphère pour le chapitre qui commençait, et, apparemment, s'éloignait tant d'une pareille esquisse lumineuse. Jamais je n'ai pris mieux conscience que le passage d'un genre litteraire a l'autre n'allait pas de soi, et qu'il fallait commencer l'apprentissage d'une autre écriture, qui avait une capacité et une ambition differentes, dans un autre champ. J'espère que les dernieres 
versions de mes romans, si j'excepte La mort vive, sont aujourd'hui plus convaincantes, avec leur spécificité propre.

***

Je serais plutôt d'accord avec Umberto Eco pour faire profession de foi envers la narrativité, malgré quelques déviations moins heureuses du récit dans le cinéma. Il me semble, avec Eco, que le roman peut donner "le courage d'affronter les grands problèmes métaphysiques», qu'il est une sorte de voie royale d'une certaine forme d'" activité philosophique", tout en liberrant le narrateur de la pesanteur, de la complexité des théories. Bien entendu, cette forme de pensée convient mieux a mon tempérament de poète. Il me faut en général saisir les idees par intuition, à travers la lumiere, le son qui traversent nos sens. Voild une forme de connaissance où le roman peut faire merveille.

$$
\text { * * * }
$$

Autant, par exemple, le détail concret dans la langue poétique permet l'arrachement de la lourdeur et de la matière ("La mer entiere se leva auprès de la table.»), autant le détail dans la prose romanesque, bien en relief, nous renforce dans une feinte habile, nous amene dans la proximité du réel pour mieux le simuler et le surmonter. Si bien que l'on arrive un court instant à ne plus faire la difference entre les êtres de fiction et les êtres de la vie réelle. Ainsi l'innocence non sans faille de l'Idiot de Dostoïevski nous interpelle, comme si on le rencontrait dans notre vie secrete. Encore faut-il que la mise en scène, en mots, la "représentation», soit parfaite.

$$
\text { *** }
$$

Il y a dans le poème une sorte d'esthétique de la vie centrifuge, une aspiration a émerger de la matière, une navigation errante dans les parages de l'indicible. Dans le roman est suggérée une esthétique de la vie centripète, un détour par l'intérieur, vers l'intérieur des choses où se forme le sens, le projet caché d'une totalité, d'une coherence ancrée dans une vision du monde.

$$
* * *
$$

Dans le poème apparaît une narration qui se tient sur l'eperon, au-dessus de l'eternité, une narration qui en quelque sorte circule immobile entre les choses pour mieux capter le non-dicible. En ce lieu, le poeme voit que la mort est une 
illusion d'absolu, le dénonce puisqu'il a la conviction de lui échapper.

Quant au roman, il relativise la mort en la saisissant dans ses actes profanateurs de dispersion, de demembrement, mais pour mieux la contourner. N'en resterait-il; après la mort des personnages, qu'une grande forme comme La guerre et la paix, qui propose une ouverture sur l'immortalité. Le ciel ne tournoie pas en vain au-dessus d'André mourant, puisqu'il l'entraîne dans le bleu de sa spirale. A vrai dire, ce qui intéresse la poésie au sens large, dans sa plénitude, c'est une capacité de maintenir vive la lumiere au sein de toute imprégnation de la mort.

\section{***}

À proprement parler, la poésie est aussi à l'aise dans le roman que dans le poeme. Elle accepte la durée nécessaire au récit romanesque, comme elle se maintient dans une tension contemplative, dans une attention extrême en formant le poème. Ce qui ne signifie pas que le roman doit se complaire dans l'ecriture poétique, bien au contraire. Celle-ci apparaît comme un masque, une tentation de fuir les vrais problemes romanesques. N'est-ce pas, alors, la meilleure façon de mimer sa forme, de "déscuvrer", puisque la narration, le plus souvent, ne peut être efficace qu'en se depouillant d'une certaine rigidite du langage? Tandis que la contraction, la forte densité, la brieveté fulgurante fondent précisément l'espace intemporel du poème.

En somme, dans le roman, il y a une intervention de la poésie dans la durée et son domaine, pour les rendre immortels; et, dans le poème, un renversement de l'éternité dans une forme glorieuse de l'être. 